\title{
Pattern Recognition Approach of Stress Wave Propagation in Carbon Steel Tubes for Defect Detection
}

\author{
Zakiah A. Halim, Nordin Jamaludin, Syarif Junaidi, and Syed Yusainee Syed Yahya
}

\begin{abstract}
The conventional stress wave signal interpretation in heat exchanger tube inspection is human dependent. The difficulties associated with accurate defect interpretations are skills and experiences of the inspector. Hence, in present study, alternative pattern recognition approach was proposed to interpret the presence of defect in carbon steel heat exchanger tubes SA179. Several high frequency stress wave signals propagated in the tubes due to impact are captured using Acoustic Emission method. In particular, one reference tube and two defective tubes were adopted. The signals were then clustered using the feature extraction algorithms. This paper tested two feature extraction algorithms namely Principal Component Analysis (PCA) and Auto-Regressive (AR). The pattern recognition results showed that the $A R$ algorithm is more effective in defect identification. Good comparisons with the commonly global statistical analysis demonstrate the effective application of the present approach for defect detection.
\end{abstract}

Index Terms-Auto-regressive, pattern recognition, principal component analysis, stress wave.

\section{INTRODUCTION}

Heat exchanger tube leakage is expensive for plant operation. It has high potential of catastrophic failure that could results in property damage and loss of life. Hence, tubes inspection is carried out at specified periodical interval as a proactive measure in monitoring and assessing the tube health and integrity. Several different approach have been developed to gain information on the tube condition. One of successful approach is interactions of stress wave propagation with the irregularities in the tube structure. Since this approach is indirect, the evaluation of recorded signals requires basic understanding involved in the physical process to infer the tube health condition [1].

Moreover, in conventional tube inspection methods, defect is typically visually interpreted by certified personnel by comparing the captured signals from inspected tubes with the signals from calibrated tube according to either time of arrival or single parameter such as amplitude and frequency level [2]. Prior knowledge of defect signal pattern and experience are essential for a reliable interpretation. This

Manuscript received March 17, 2014; revised May 10, 2014. The materials for this work was supported by Tenaga Tiub Sdn. Bhd. A.H. Zakiah thanks the Ministry of Education (Higher Education) Malaysia and Universiti Teknikal Malaysia Melaka (UTeM) for financial support of her study.

A. H. Zakiah is with the Universiti Teknikal Malaysia Melaka, 76100 Melaka, Malaysia (e-mail: zakiahh@utem.edu.my).

N. Jamaludin and J. Syarif are with the Universiti Kebangsaan Malaysia, 43600 Bangi, Malaysia (e-mail: nordin@eng.ukm.my, syarif@eng.ukm.my).

S. Y. S. Yahya is with the Universiti Teknologi MARA, 40450 Shah Alam, Malaysia (e-mail: syedy237@salam.uitm.edu.my). requires adequate training hours to develop the expertise and competency. A large sum of resources is required for proper training, but more often being neglected because of limited fund [3].

Driven by the demand for higher performance and faster industrial production, advancing trends in automated signal interpretation are expected. One suitable approach to autonomous interpretation is the application of feature extraction based pattern recognition techniques. Previous studies are found to be successfully employed pattern recognition techniques to interpret the hidden trends of the complex stress wave signals [4]-[7]. Additionally, pattern recognition has successfully facilitated autonomous defect identification process in many structural application such as bearing, composite beam and pressure vessel [8]-[10].

Hence, the aim of this paper is to explore an alternative to signal interpretation using pattern recognition approach, which was not considered in these earlier studies. The present work compares two feature extraction algorithms, namely Principal Component Analysis (PCA) and Auto-Regressive (AR) to distinguish high frequency stress wave signal data from Vibration Impact Acoustic Emission (VIAE). VIAE was carried out on three similar dimension and material, with exceptional of presence of artificial defect in each of the tube. The findings of this study demonstrated the potential of pattern recognition approach and its suitability for signal interpretation is investigated. The current study will help the inspector to gain better insight on the stress wave signal pattern in the presence of defect and thus, improve defect assessment.

\section{Pattern ReCognition APPROACH}

The objective of pattern recognition is to obtain the ideal patterns based on specified factor and thus split them into distinct groups. Generally, there are three major stages involves in pattern recognition, which are: i) preprocess of the stress wave signals, ii) clustering and iii) validation of formed clusters. The primary step in pattern recognition is preprocessing includes filtering and feature selection based on selected preprocessing algorithm. In current study, the feature extraction algorithms undertaken are PCA and AR in the attempt to speedy the signal interpretation. PCA has been successfully used in classifying the signals from different sources [11]-[13]. This algorithm determined the covariance value that represent relationship between each signal. Covariance is a measure between 2 dimensions $(X$ and $Y$ ) is calculated using (1). If the data has more than 2 dimensions, there will be more than one covariance to be calculated. For an $n$-dimensional data set, the number of covariance, $n_{c v}$ 
values can be calculated is given by (2):

$$
\begin{gathered}
\operatorname{Covariance}(X, Y)=\frac{\sum_{i=1}^{n}\left(X_{i}-\bar{X}\right)\left(Y_{i}-\bar{Y}\right)}{n-1} \\
n_{c v}=\frac{n !}{(n-2) ! \times 2}
\end{gathered}
$$

Structural differences between these observed signals will be obtained through decomposition of eigenvalues and eigenvectors. The sequential of PCA algorithm is illustrated in the flowchart as in Fig. 1.

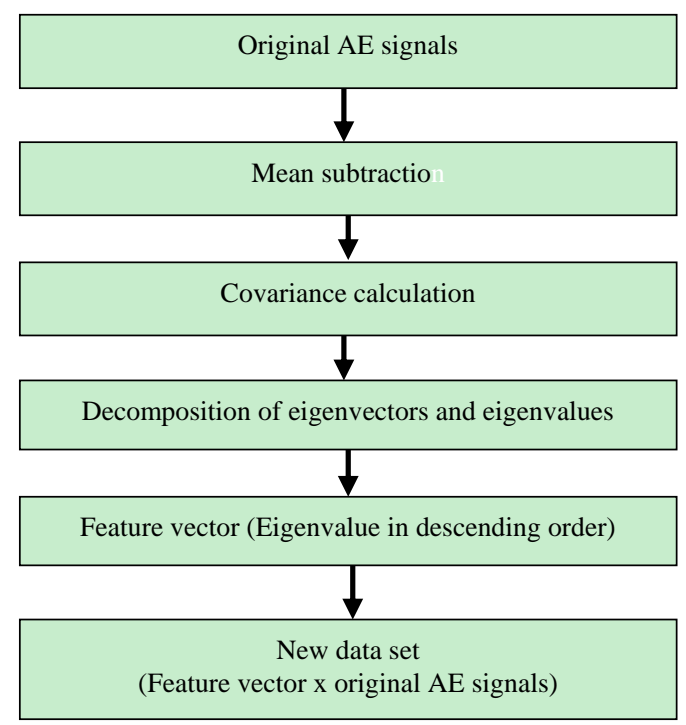

Fig. 1. PCA sequential procedure.

Marec et al. [11] has exploited PCA coefficients to identify the damage mechanisms in polymer-based composite. Stress waves associated with damage mechanisms were effectively discriminated the damage mechanisms. Besides, Holford [12] have applied PCA to several groups of acoustic emission (AE) signals radiating from different defect source in aircraft structures. The results of the analysis show that AE signals from defects in the landing gear were successfully isolated in the different cluster. In different studies, Omid et al. [13] were able to separate pistachio nuts according to their sizes and condition by taking advantage of this extraction algorithm. In the study, stress wave signal emitted from a kernel when hitting a steel plate can be properly classified according to different nut condition.

Similarly, AR coefficients have been used by other researchers including Jamaludin and Mba [14], [15] and Atamturktur et al. [16] to classify stress wave signatures. A stationary random process $x(n)$ may be described as an AR model of order $M$ is expressed as:

$$
x(n)=\sum_{k=1}^{M} h(k) \times(n-k)+W(n)
$$

in which $W(n)$ is a white noise process of zero mean and variance $\sigma^{2}, h(k)$ is the AR coefficients and $\mathrm{M}$ is the model order. Works by Jamaludin and Mba [14], [15] involved the study of bursts associated with low speed machinery. They have employed Levinson-Durbin's forward linear prediction algorithm to solve (3) to represent the shape of the bursts by a set of AR coefficients. Stress waves signatures associated with several simulated mechanical defects normally encountered in a roller bearing were successfully classified using cluster analysis technique. In another study, Jamaludin and $\mathrm{Mba}$ [15] also extended the application of AR coefficients to successfully identify defects in real roller bearings. In addition, Atamturktur et al. [16] has demonstrated that AR algorithm show great achievement in detecting structural separation in historic masonry.

Subsequently, the clustering is made based on basic and incremental distance value of the preprocess feature using hierarchical procedure or K-means [17], [18]. Then, the most similar clusters that have the smallest distance combine into a new cluster. Hierarchical clustering procedure are often preferred when discovering the natural pattern of the data. Accurate interpretation of the clusters involves examining each component in the cluster and allocate label that representing the nature of the cluster. As the final stage, validation is required to ensure the clustering results represent the general population or has practical significance.

The main advantage of pattern recognition is that it executes classification without having any idea about the distribution of the measurements [17]. Albeit huge mass of stress wave data analysed by employing pattern recognition, the right patterns are identified, and any outliers is able to be spotted. Hence, pattern recognition approach can be regarded as suitable to automate the signal interpretation of measurement for defect detection. This attempt is suitable for the current circumstance that requires effective condition classification.

\section{METHODOLOGY}

\section{A. Tube Description}

Heat exchanger steel tubes A179 were used for the inspection using integrated vibration and acoustic emission (AE) systems called VIAE. These $1 \mathrm{~m}$ long tubes have a $19.05 \mathrm{~mm}$ outside diameter and $2.11 \mathrm{~mm}$ wall thickness. The measurement of stress wave was performed on three steel tubes $\mathrm{R}$, Tube 1 and Tube $2 . \mathrm{R}$ is the reference tube, and Tube 1 and Tube 2 are the defective tubes. The defect is a $1 \mathrm{~mm}$ diameter hole artificially crafted by electrical discharge machining at $100 \mathrm{~mm}$ from one of the tube ends. Tube 1 has $40 \%$ wall loss depth defect and Tube 2 is through-hole defect. Both tube ends were fixed supported as illustrated in Fig. 2.

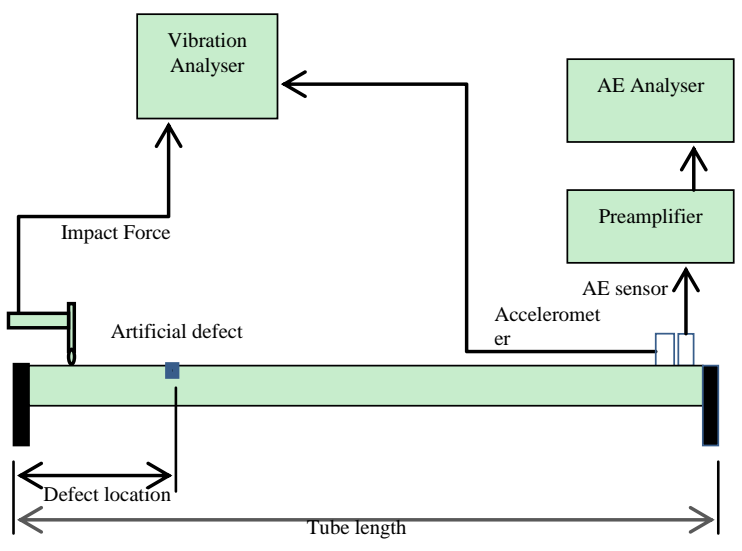

Fig. 2. Experimental set up in laboratory environment. 


\section{B. Testing Procedure}

The impact was repeated for 20 times for each tube condition. Input force load and coherency plot are retrieved from PULSE Labshop. The impact force load represent the magnitude of force impacted on the tube. A typical time history of the applied force load on the tubes is shown in Fig. 3(a). Distinctively, Fig. 3(a) clearly showed that the typical impact has a time delay of $18 \mathrm{~ms}$ and features a sharp peak of $37 \mathrm{~N}$. The consistency of the repeated impact was validated using coherency plot. The coherency plot represents the linearity of the impact loads on the tube. The coherence plot illustrated in Fig. 3(b) verified that a uniform impact force load has been applied to the tubes.

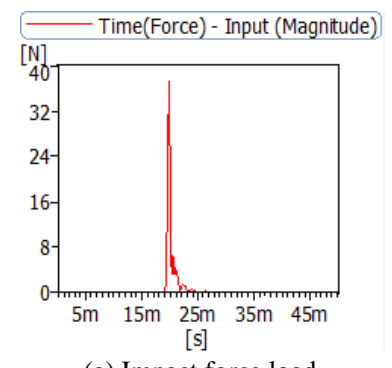

(a) Impact force load.

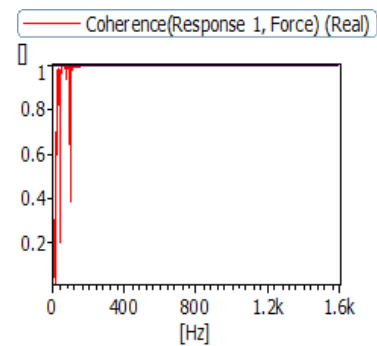

(b) Coherence plot.
Fig. 3. Typical vibration data.

The impact by hammer initiated stress wave propagation in the tube. In VisualAE software, 20 time history of stress wave signals were recorded for each impact in each tube condition. Each stress wave signals for reference tube was labelled as R. Stress wave signals from the defective tubes Tube 1 and Tube 2 were labelled as T1 and T2, respectively.

The stress wave signals acquired by the high frequency transducer were analysed using PCA and AR algorithms. Having calculated the appropriate covariance or AR coefficients associated with each signals, the pattern recognition analysis was undertaken using hierarchical clustering. The cluster analysis is undertaken to classify similar signals into similar groups. Several methods are possible, with each of specific similarity characteristic. The most recognized measure of similarity distance is Euclidean distance, or also referred to as straight-line distance [19]. In this study, the Euclidean distance is exploited as measures of similarity. The hierarchical clustering measures the Euclidean distance between the covariance or AR coefficients associated with each signals. The similarity is calculated between each of the signals to enable each signal to be compared with each other. Smaller distances signified the signals have greater similarity.

Then, Ward's method is employed as the agglomerative algorithm. The signals with minimum sum of square are grouped together. The sum of squares of the distances between all signals in the cluster is recalculated, and the signals with the minimum sum of square to the newly formed group are clustered with it. This step is repeated every time a signal is added to the cluster until all the signals are grouped. Finally, the validation is done to check whether the algorithm produced the correct cluster. The results were illustrated using dendrogram, with the nearest Euclidean distances are clustered together. The resulted clustering is illustrated using dendrogram. The computing software used was MATLAB.

\section{RESUlTS AND DisCUSSION}

\section{A. Time History}

The typical high frequency stress wave propagated along the tubes are obtained when the tube is impacted on one of tube ends. Fig. 4 illustrates time waveforms of propagated stress wave in the three inspected tubes. Generally, the typical stress wave represents multiple repetitive burst signal prior to complete attenuation. Each burst has approximately $250 \mu$ s period length. In addition, the shape and characters of reference tube $\mathrm{R}$ and defective tubes Tube 1 and Tube 2 are distinctly different due to different propagation path. It is clearly shown that Tube 1 and Tube 2 has additional burst signals compared to R. Since Tube 1 and Tube 2 have artificial defect that obstruct the stress wave propagation, more reflection of the stress wave from the original propagation path is observed. As illustrated in Fig. 4(a), the stress wave in reference tube $\mathrm{R}$ completely attenuated at $4542 \mu \mathrm{s}$. Meanwhile, the stress wave in defective tubes Tube 1 and Tube 2 completely attenuated at longer period of $7262 \mu \mathrm{s}$ and $8674 \mu \mathrm{s}$, as shown in Fig. 4(b) and Fig. 4(c) respectively.

Based on stress wave propagation theory, the different waveforms are attributed to different stress wave propagation path. From the above findings, it appears that the presence of defect modifies the stress wave propagation path in the tube structure. The artificial defect caused more reflection of stress wave, diffraction, beam spreading and surface waves, which effected the stress wave to attenuate at longer period and have more multiple burst. The deeper the defect depth, more burst attenuation of stress wave is observed. By inspection, it is obvious that defective signals appear to be more complex than the reference tube $\mathrm{R}$ signals. Each tube has a distinct stress wave signal. However, actual physical interpretation of the stress wave signals is more difficult, especially as no obvious differences in the amplitude levels and the burst repetitions of the two defective tubes are almost identical.

\section{B. Cluster Analysis}

The works presented here has focused on the use of the PCA and AR algorithms, which takes advantage of feature extraction analysis of data to classify the stress wave signals by employing hierarchical clustering. Only 100,000 data point from the stress wave history is considered in the cluster analysis as it is observed that the stress wave is completely attenuated by $0.01 \mathrm{~s}$. The purpose of cluster analysis is to put together similar characteristic into a group and dissimilar characteristic into another group, to meet the objective of pattern recognition. Each and every group has unique characteristics that differentiate one from another. The achieved clustering results are illustrated using dendrogram plot as demonstrated in Fig. 5 and Fig. 6. The x-axis represents the Euclidean distances between the input values. The $y$-axis represents the stress wave signals. The stress wave signals by label R, T1 and T2 represented stress wave signals of reference tube, defective tube $\mathrm{T} 1$ and defective tube $\mathrm{T} 2$, respectively.

PCA is a statistical technique that involved variable reduction procedure. The relationship between the signals 
was determined by exploiting covariance matrix. It is achieved through decomposition of covariance matrix into eigenvalue and eigenvector. The hidden pattern in the signals is observed at first principal component (PC). Typically the first $\mathrm{PC}$ has eigenvalue greater than 1. Each signals contributes one unit of variance to the total variance in the data set. Any component that displays an eigenvalue greater than 1 is accounting for a greater amount of variance, while the later components account for relatively smaller amounts [19].

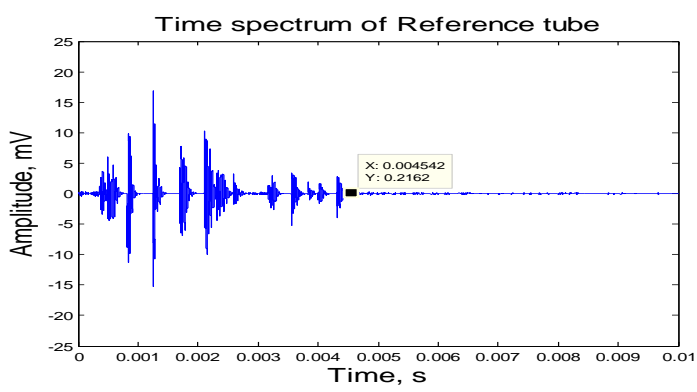

(a) Reference tube $\mathrm{R}$

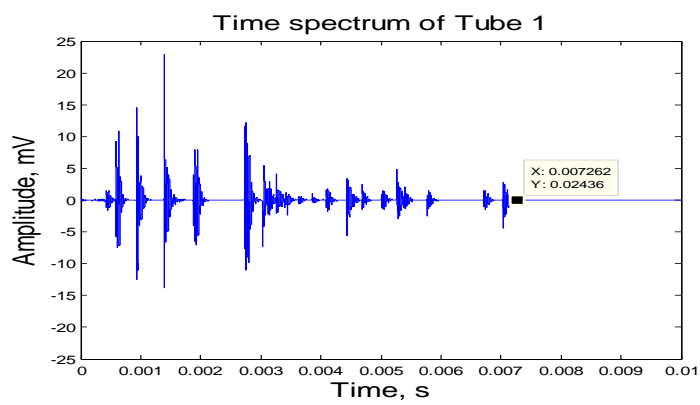

(b) Tube 1 .

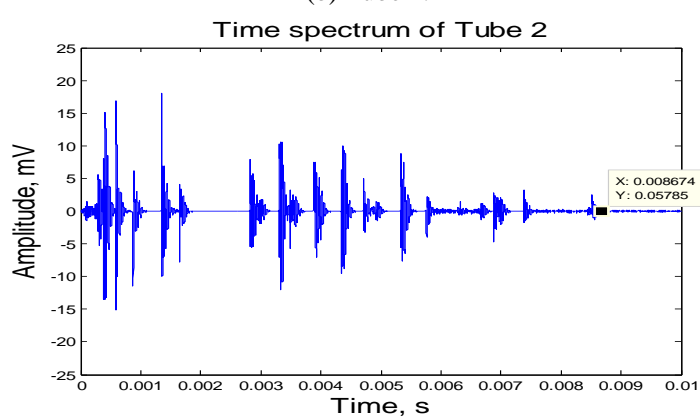

(c) Tube 2

Fig. 4. Time history produces by force impact at the tube end of the carbon steel tubes.

Application of PCA to the stress wave signals resulted two major groups as demonstrated in Fig. 5. However, it was examined in Fig. 5 that major distinction between the stress wave signals of reference tube $\mathrm{R}$ and defective tubes Tube 1 or Tube 2 were not achieved using PCA algorithm. It can be identified from Fig. 5(a) that 12 of stress wave signals associated with Tube 1 signals were merged with all the stress wave signals associated with reference tube in Cluster 1 whereas only 8 of stress wave signals associated with Tube 1 were grouped in Cluster 2. Similar outcome was observed when clustering stress wave signals from reference tube and Tube 2. Cluster 1 and Cluster 2 consist of combination of stress wave signals associated with reference tube and Tube 2 as portrayed in Fig. 5(b). It was clearly evident that clustering results using PCA approach did not result in any clear grouping, thus becomes inappropriate for pattern recognition of stress wave propagation in steel tube.

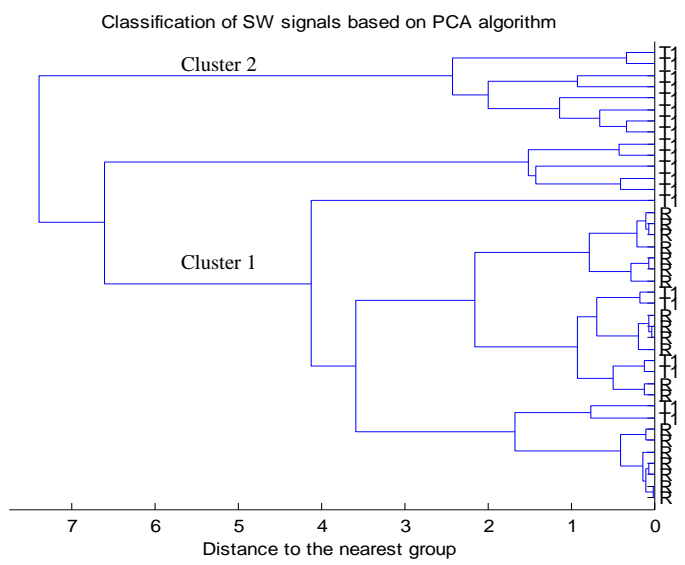

(a) Reference tube vs Tube 1 .

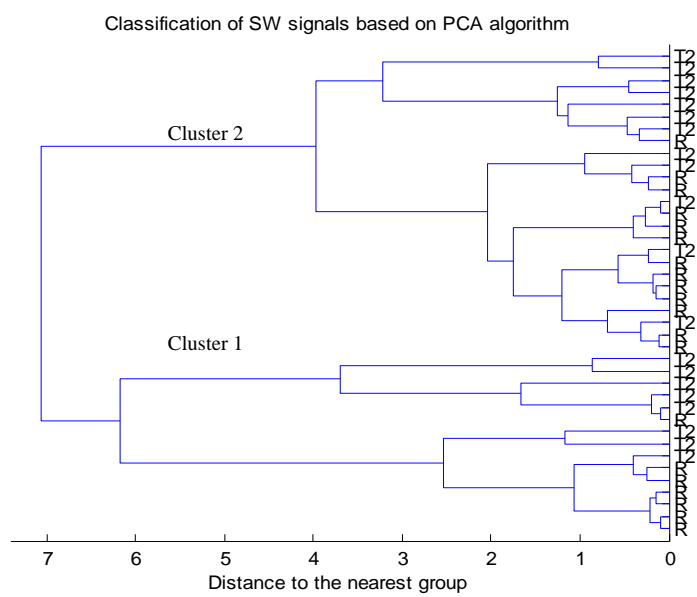

(b) Reference tube vs Tube 2 .

Fig. 5. Classification analysis using Principal Component Analysis algorithm.

The lack of success in discriminating between the stress wave signals was observed due to PCA as a statistical pattern recognition. Similar peak amplitude was observed from time history of stress wave propagation (see Fig. 4) for all the three tubes, which resulted in a statistically similarity in variance between all the measured stress wave. Consequently, small variance between these observed signals caused no characteristic differences. Thus, all the signals were mixed up and show lack of group structure when employing PCA algorithm.

Conversely, AR approach is utilized due to the ability of the AR coefficients to identify and classify burst type signals based on their shape feature. The shape is represented by AR coefficients. Application of Levinson-Durbin's forward prediction error on the stress wave signals indicated that a 20th model order was sufficient to represent the stress wave signals. Every signal is represented by a $21 \mathrm{AR}$ coefficients (20th AR model order). Subsequently, the hierarchical clustering method described in Section III was applied to group the signals.

It is noticeable in Fig. 6 that pattern recognition of stress wave signals using AR algorithm resulted into two major clusters. Primarily, two major groups separate between the reference tube and defective tube T1 signals. The first cluster comprises of stress wave signals of reference tube $\mathrm{R}$ and the second cluster contains stress wave signals of defective tube 
T1 as illustrated in Fig. 6(a). Classifying stress wave signals from reference tube and Tube 2 produced identical result. All signals associated with reference tube $\mathrm{R}$ are clustered into Cluster 1 and signals associated with $\mathrm{T} 2$ are assembled in Cluster 2 as depicted in Fig. 6(b). The separation between the reference tube $\mathrm{R}$ signals and defective tubes $\mathrm{T} 1$ and $\mathrm{T} 2$ signals are clearly pronounced. In all cases different cluster groups of $\mathrm{R}$ signals and defective tubes $\mathrm{T} 1$ and $\mathrm{T} 2$ signals were clearly distinct, with the Euclidean distances between the clusters was in excess of 0.4. It is clear that reasonable good defect signals has been attained using AR algorithm. The two cluster groups associated with tube condition are attributed to the different stress wave propagation path. Defective tubes have longer attenuation time, which resulted to different shape feature compared to reference tube.

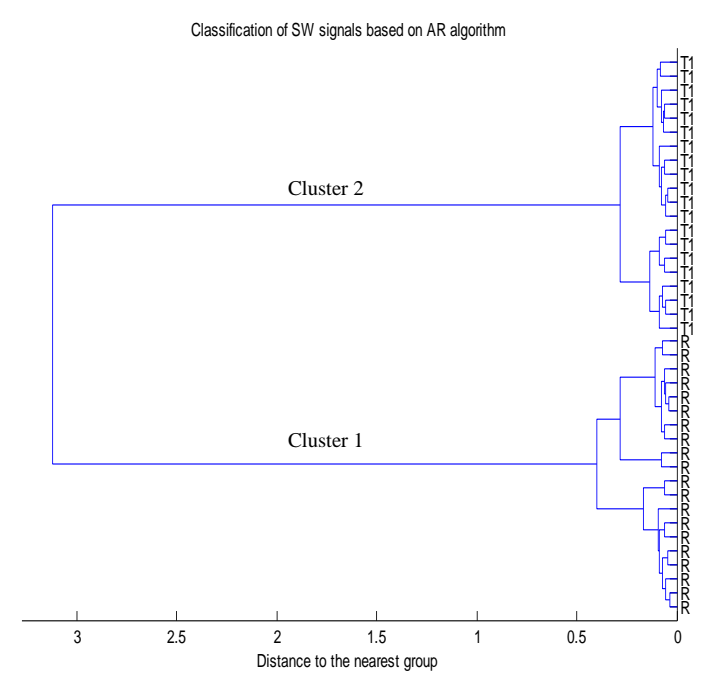

(a) Reference tube vs Tube 1

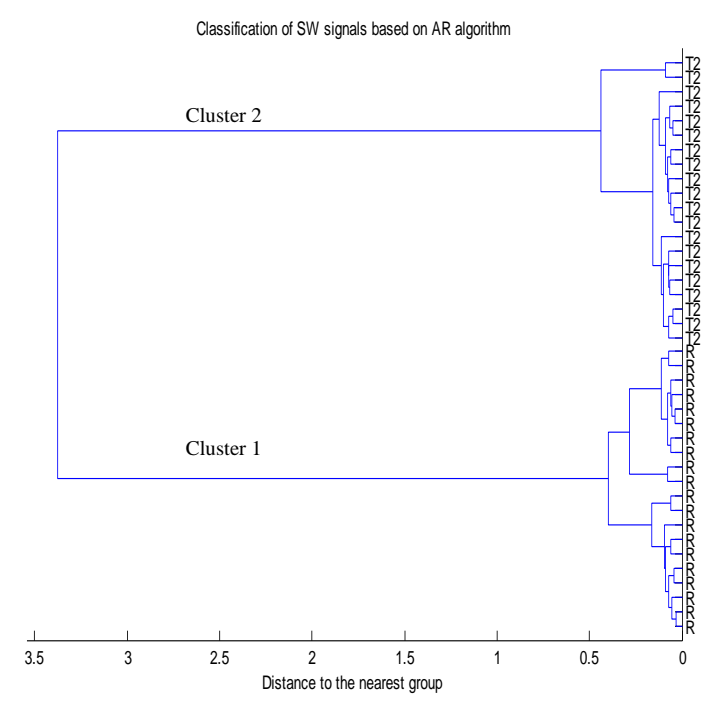

(b) Reference tube vs Tube 2

Fig. 6. Classification analysis using Auto-regressive algorithm.

It was evident that AR cluster technique showed distinct separations between the stress wave signals. As introduced in Section I, the aim of the application of pattern recognition in stress wave captured from VIAE feature extraction is the identification of defect signal. Hence, it is postulated that stress wave classification using AR algorithm is more suitable than the established PCA algorithm. Given the scenario of the tube with a defect, it is probable that the stress wave captured from VIAE method could be successfully differentiated using AR algorithm. Thus, clustering results yielded distinct cluster groups from the reference signals as demonstrated in Fig. 6, this would be evident for the presence of defect in the tube. If no distinct groups were evident, then the tube would be passed a defect free. Application of PCA algorithm would generally mislead the interpretation results as no clearly defined groups as shown in Fig.5, which will be interpreted as defect free, when the actual is not the case. The present finding using AR algorithm is consistent with stress wave data when assessed using global statistical analysis [20].

\section{CONCLUSION}

The application of two feature extraction algorithms have been demonstrated. AR algorithm successfully distinguish the defective signals pattern than PCA algorithm. The dendrogram enable a clear division of signal wave and enable quick and easy interpretation. The application of feature extraction using AR algorithm reveals meaningful information in the stress wave data sets from VIAE methods. This findings provide insights that can be useful to meet the unique demands of the rapidly evolving industry so that the inspector is able to accurately identify the defect and at the same time evaluate the tube health effectively.

\section{REFERENCES}

[1] Y. Lin, C. Chang, S. F. Kuo, and H. C. Liou, "A simple device for detecting impact time in impact-echo testing of concrete," NDT E Int., vol. 37, pp. 1-8, Jan. 2004.

[2] J. J. Da Silva, A. M. N. Lima, F. H. Neff, and J. S. R. Neto, "Non-invasive fast detection of internal fouling layers in tubes and ducts by acoustic vibration analysis," IEEE Trans. on Instrum. Meas., vol. 58, pp. 108-114, 2009.

[3] N. J. Carino, "Training: Often the missing link in using NDT methods," Constr. Build. Mater., vol. 38, pp. 1316-1329, Jan. 2013.

[4] C. Patapy, A. Proust, D. Marlot, M. Huger, and T. Chotard, "Characterization by acoustic emission pattern recognition of microstructure evolution in a fused-cast refractory during high temperature cycling," J. Eur. Ceram. Soc., vol. 30, pp. 3093-3101, Nov. 2010.

[5] W. Qian, L. Xie, D. Huang, and X. Yin, "Pattern recognition of fatigue damage acoustic emission signals," in Proc. International Conference on Mechatronics and Automation, 2009, pp. 4371-4375.

[6] C. K. Liew and M. Veidt, "Pattern recognition of guided waves for damage evaluation in bars," Pattern Recognition Letters, vol. 30, pp. 321-330, 2009.

[7] F. Schwenker and E. Trentin, "Pattern classification and clustering: A review of partially supervised learning approaches," Pattern Recognition Letters, vol. 37, pp. 4-14, 2014.

[8] M. Elforjani and D. Mba, "Accelerated natural fault diagnosis in slow speed bearings with Acoustic Emission," Eng. Fract. Mech., vol. 77, pp. 112-127, Jan. 2010.

[9] S. E. Hamdi, A. Le Duff, L. Simon, G. Plantier, A. Sourice, and M Feuilloy, "Acoustic emission pattern recognition approach based on Hilbert-Huang transform for structural health monitoring in polymer-composite materials," Appl. Acoust., vol. 74, pp. 746-757, May 2013.

[10] L. Li, W. Zhang, Z. Chen, L. Wen, and F. Wang, "Application of pattern recognition to the acoustic emission signals of carbon steel," in Proc. $2^{\text {nd }}$ International Congress on Image and Signal Processing, 2009, pp. 1-4.

[11] A. Marec, J.-H. Thomas, and R. El Guerjouma, "Damage characterization of polymer-based composite materials: Multivariable analysis and wavelet transform for clustering acoustic emission data," Mech. Syst. Signal Process., vol. 22, pp. 1441-1464, Aug. 2008. 
[12] K. M. Holford, R. Pullin, S. L. Evans, M. J. Eaton, J. Hensman, and K. Worden, "Acoustic emission for monitoring aircraft structures," in Proc. the Institution of Mechanical Engineers, Part G: Journal of Aerospace Engineering, 2009, pp. 525-532.

[13] M. Omid, A. Mahmoudi, and M. H. Omid, "Development of pistachio sorting system using principal component analysis (PCA) assisted artificial neural network (ANN) of impact acoustics," Expert Syst. Appl., vol. 37, pp. 7205-7212, Oct. 2010.

[14] N. Jamaludin and D. Mba, "Monitoring extremely slow rolling element bearings: part I," NDT E Int., vol. 35, pp. 349-358, Sep. 2002.

[15] N. Jamaludin and D. Mba, "Monitoring extremely slow rolling element bearings: part II," NDT E Int., vol. 35, pp. 359-366, Sep. 2002.

[16] S. Atamturktur, L. Bornn, and F. Hemez, "Vibration characteristics of vaulted masonry monuments undergoing differential support settlement," Eng. Struct., vol. 33, pp. 2472-2484, Sep. 2011.

[17] V. Kostopoulos, P. Tsotra, P. Karapappas, S. Tsantzalis, A. Vavouliotis, T. H. Loutas, A. Paipetis, K. Friedrich, and T. Tanimoto, "Mode I interlaminar fracture of CNF or/and PZT doped CFRPs via acoustic emission monitoring," Compos. Sci. Technol., vol. 67, no. 5, pp. 822-828, Apr. 2007.

[18] M. G. R. Sause, A. Gribov, A. R. Unwin, and S. Horn, "Pattern recognition approach to identify natural clusters of acoustic emission signals," Pattern Recognit. Lett., vol. 33, no. 1, pp. 17-23, 2012.

[19] T. Rajalahti and O. M. Kvalheim, "Multivariate data analysis in pharmaceutics: a tutorial revie," Int. J. Pharm., vol. 417, pp. 280-90, Sep. 2011.

[20] A. H. Zakiah, N. Jamaludin, J. Syarif, and S. Y. S. Yahya, "Detection and analysis of defect in steel using vibration impact acoustic emission (VIAE) method," Int. Rev. Mech. Eng., vol. 8, no. 1, pp. 277-282, 2014.

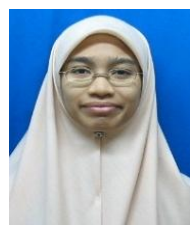

A. H. Zakiah studied mechanical engineering (M.EngSc.) at the University of New South Wales (UNSW), Australia in 2006 and started her career at Universiti Teknikal Malaysia Melaka (UTeM) as a lecturer.

She is currently pursuing her $\mathrm{PhD}$ study in Universiti Kebangsaan Malaysia (UKM). Her current research interest are in the area of structural integrity, non-destructive testing method such as acoustic emission and evaluation of defect behavior from signal processing analysis.

Mrs. Zakiah is a member of Malaysian Society of Non Destructive Testing (MSNT) and has Malaysian Skills Certificate as Ultrasonic Tester Level 2.

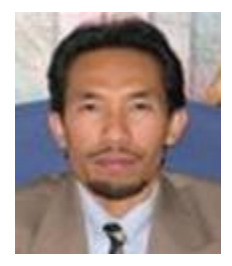

N. Jamaludin is currently the program coordinator for Industrial Training (Mechanical) Program in Faculty of Engineering and Built Environment. After earning his $\mathrm{PhD}$. degree in 2000 in the area of condition monitoring at Cranfield University, he continued his career as a lecturer in Universiti Kebangsaan Malaysia (UKM) until promoted to his current position.

$\mathrm{He}$ has more than 13 years of experience in the condition monitoring field. Area of research are investigations on material damage mechanism in structural and machinery components using acoustic emission and metal magnetic memory technology as part of integrity monitoring analysis and assessment.

Assoc. Prof. Dr. Jamaludin is a fellow in Welding Institue of Malaysia, Chairman of Malaysian Acoustic Emission Working Group as well as member of Malaysian Society of Non Destructive Testing (MSNT)

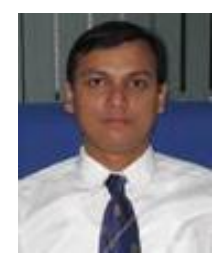

J. Syarif is an associate professor at Faculty of Engineering and Built Environment, Universiti Kebangsaan Malaysia (UKM). He earned his PhD. Degree from Kyushu University, Japan in 2003. He is the receipient of Monbukagakusho Scholarship from 1998-2000 and 2000-2003 for his Master and PhD degrees.

$\mathrm{He}$ has more than 10 years experience in structural material engineering and properties, physical metalurgy as well as alloy design and has published approximately 47 papers in the respective field.

Assoc. Prof. Dr. Syarif is a member of Iron and Steel Institute of Japan Institute of Materials, Malaysia and Malaysian Powder Metallurgy and Particulate Materials Association.

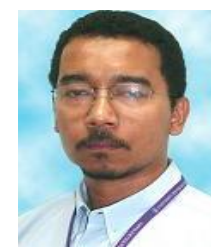

S. Y. S. Yahya is a senior lecturer at Faculty of Applied Science, Universiti Teknologi MARA and is teaching about Material Technology. He obtained his $\mathrm{PhD}$ degree in advance materials from Universiti Kebangsaan Malaysia (UKM) in 2005.

His research expertise are in materials physics and advanced materials.

Dr. Yahya is a member of Malaysia Society of Non Destructive Testing (MSNT) and American Society of Non Destructive Testing (ASNT). 